\title{
Opisthorchis viverrini: efficacy and tegumental alterations following administration of tribendimidine in vivo and in vitro
}

\author{
Jennifer Keiser • Jürg Utzinger • Shu-Hua Xiao • \\ Peter Odermatt $\cdot$ Smarn Tesana
}

Received: 21 November 2007 / Accepted: 30 November 2007 / Published online: 3 January 2008

(C) Springer-Verlag 2007

\begin{abstract}
The tegumental changes in adult Opisthorchis viverrini induced by tribendimidine were analyzed by scanning electron microscopy (SEM). We exposed $O$. viverrini to tribendimidine at a concentration of $10 \mu \mathrm{g} /$ $\mathrm{ml}$ for $4 \mathrm{~h}$. In addition, hamsters were treated with a single $400 \mathrm{mg} / \mathrm{kg}$ oral dose of tribendimidine and flukes were recovered from the bile ducts $24,48,72$, and $96 \mathrm{~h}$ posttreatment. SEM analysis of the flukes incubated in vitro showed only mild damage of the tegument. Twenty-four hours post-treatment of hamsters, extensive disruption such as sloughing, furrowing, or blebbing of the tegument was evident, which did not increase in severity $48-72 \mathrm{~h}$ posttreatment. Ninety-six hours after tribendimidine administration, the majority of flukes had been expelled. A single $400 \mathrm{mg} / \mathrm{kg}$ oral dose of tribendimidine administered to $O$. viverrini-infected hamsters yielded a worm burden
\end{abstract}

J. Keiser $(\bowtie)$

Department of Medical Parasitology and Infection Biology, Swiss Tropical Institute,

P.O. Box, CH-4002 Basel, Switzerland

e-mail: jennifer.keiser@unibas.ch

J. Utzinger $\cdot$ P. Odermatt

Department of Public Health and Epidemiology,

Swiss Tropical Institute,

P.O. Box, CH-4002 Basel, Switzerland

S.-H. Xiao

National Institute of Parasitic Diseases,

Chinese Center for Disease Control and Prevention,

200025 Shanghai, People's Republic of China

\section{S. Tesana}

Department of Parasitology, Faculty of Medicine,

Khon Kaen University,

Khon Kaen, Thailand reduction of $63.0 \%$. In conclusion, our experiments confirm that tribendimidine possesses interesting trematocidal properties, which warrant additional investigations and provide further data for subsequent clinical trials.

\section{Introduction}

Opisthorchis viverrini, a liver fluke belonging to the foodborne trematodes, is the causative agent of opisthorchiasis. At present, an estimated 67.3 million people are at risk and 9 million are infected with the highest prevalence found in Cambodia, Lao People's Democratic Republic, Thailand, and Viet Nam. The most serious complication of opisthorchiasis is cholangiocarcinoma, the bile duct cancer (Haswell-Elkins and Levri 2003; Sripa 2003; Keiser and Utzinger 2007b). Since the early 1980 s, when the good safety and therapeutic profile of praziquantel against opisthorchiasis had been established, treatment and control of opisthorchiasis relies on this drug (Keiser and Utzinger 2007b). Although treatment failures have not yet been reported for praziquantel in O. viverriniinfected patients, the search for alternative trematocidal drugs is warranted. A promising strategy to develop novel drugs for neglected tropical diseases, in this case foodborne trematodiasis, is based on label extensions of existing drugs (Nwaka and Hudson 2006). Hence, evaluating the trematocidal potential of drugs that are already on the market for related diseases is a key approach for our own drug discovery and development portfolio at the Swiss Tropical Institute (Keiser and Utzinger 2007a).

Tribendimidine is a derivative of amidantel, synthesized and developed in China (Ren et al. 1987). Laboratory and clinical investigations demonstrated that this drug is safe and has a broad spectrum of activity against nematodes that 
are of considerable public health significance (e.g., Ascaris lumbricoides, Enterobius vermicularis, and the hookworms) (Xiao et al. 2005). New research has shown that tribendimidine is also active against Strongyloides ratti, in particular the adult stages (Keiser et al. 2008). Importantly, in recent laboratory investigations we could extend activity from nematodes to trematodes. For example, worm burden reductions of $100 \%$ were achieved with a single $125-\mathrm{mg} / \mathrm{kg}$ oral dose of tribendimidine against the intestinal trematode Echinostoma caproni harbored in mice (Keiser et al. 2006c). High worm burden reductions were also achieved with single oral doses of tribendimidine against Clonorchis sinensis in the rat and $O$. viverrini in the hamster (Keiser et al. 2006a).

The aim of this study was to further clarify the opisthorchicidal properties of tribendimidine. We first evaluate the therapeutic efficacy of tribendimidine after a single oral dose administered to hamsters infected with adult $O$. viverrini. Next, we assessed tegumental alterations in adult $O$. viverrini recovered from treated hamsters using a scanning electron microscope (SEM). Finally, we investigated drug-induced tegumental alterations of $O$. viverrini after in vitro exposure to tribendimidine.

\section{Materials and methods}

\section{Tribendimidine}

Tribendimidine was obtained from the Department of Pharmaceutics, National Institute of Parasitic Diseases, Chinese Center for Disease Control and Prevention (Shanghai, China). The drug was prepared in homogenous suspensions in $7 \%$ Tween- 80 and $3 \%$ ethanol before oral administration. For the in vitro studies, a $10-\mathrm{mg} / \mathrm{ml}$ stock solution of tribendimidine was prepared in $60 \%$ DMSO $(v / v)$.

Ethical clearance and $O$. viverrini-hamster model

All animal studies presented here were approved by the local government of Basel, according to Swiss national regulations.

Metacercariae of $O$. viverrini were obtained from cyprinoid fish caught in Khon Kaen province, Thailand, as described previously (Keiser et al. 2006a).

Fifteen female Syrian Golden hamsters, aged 3 weeks with a weight of $\sim 100 \mathrm{~g}$, were purchased from Charles River (Sulzfeld, Germany). Animals were kept in groups of four in environmentally controlled conditions (temperature $\sim 25^{\circ} \mathrm{C}$, humidity $\sim 70 \%, 12$-h light and 12 -h dark cycle) and acclimatized for 1 week. Hamsters had free access to water and rodent diet. The hamsters were each infected intragastrically with $45 \mathrm{O}$. viverrini metacercariae.
In vivo efficacy

Four weeks post-infection, five hamsters were treated orally with a single $400-\mathrm{mg} / \mathrm{kg}$ dose of tribendimidine. Six hamsters were left untreated and served as controls. Hamsters were killed and dissected 2 weeks post-treatment. $O$. viverrini were removed from the liver, gall bladder, and bile ducts and counted. We used the Kruskal-Wallis test $(\mathrm{KW})$ to test for statistical significance of the median worm counts between the treatment and control group. Statistical significance was considered at a level of 5\%. We used version 2.4.5 of Statsdirect (Statsdirect Ltd; Cheshire, UK) for statistical analysis.

In vitro SEM observations

Opisthorchis viverrini obtained from untreated control animals were rinsed in warm $0.9 \%(w / v) \mathrm{NaCl}$ and six flukes were incubated in $2-\mathrm{ml}$ minimal essential medium (MEM) (Gibco), supplemented with antibiotics $(50 \mu \mathrm{g} / \mathrm{ml}$ streptomycin plus $50 \mathrm{U} / \mathrm{ml}$ penicillin) (Gibco). O. viverrini were incubated in a medium containing $10 \mu \mathrm{g} / \mathrm{ml}$ of tribendimidine. Controls were prepared by incubating flukes in MEM supplemented with antibiotics. Cultures were kept at $37^{\circ} \mathrm{C}$ in an atmosphere of $5 \% \mathrm{CO}_{2}$ and observed after exposure for $4 \mathrm{~h}$ under a dissecting microscope. Subsequently, all flukes were collected and processed for SEM as described below.

\section{In vivo SEM observations}

Four hamsters were treated intragastrically with tribendimidine at a single $400-\mathrm{mg} / \mathrm{kg}$ dose 5 weeks post-infection. Twentyfour, 48, 72, and $96 \mathrm{~h}$ post-treatment, one hamster each was euthanized by $\mathrm{CO}_{2}$, and flukes were collected from the bile ducts. O. viverrini from untreated hamsters served as control. The flukes were washed in saline solution and fixed with $2.5 \%$ glutaraldehyde in a phosphate-buffered saline (PBS) solution for $24 \mathrm{~h}$ at room temperature. After rinsing with PBS, the flukes were washed with distilled water, dehydrated, and critically point-dried (Bomar SPC-900; Tacoma, WA, USA). After sputter coating with gold of $20 \mathrm{~nm}$ (Baltec Med 020; Tucson, AZ, USA), the flukes were mounted on aluminum stubs and observed in a high-resolution SEM (Philips XL30 ESEM; Eindhofen, the Netherlands) at an accelerating voltage of $5 \mathrm{kV}$.

\section{Results}

In vivo drug efficacy

The effect of tribendimidine on adult $O$. viverrini harbored in hamsters, as assessed by worm burden reduction, is 
Table 1 Effect of tribendimidine against adult $O$. viverrini

\begin{tabular}{lllllll}
\hline Treatment & $\begin{array}{l}\text { Dose } \\
(\mathrm{mg} / \mathrm{kg})\end{array}$ & $\begin{array}{l}\text { No. of hamsters } \\
\text { investigated }\end{array}$ & $\begin{array}{l}\text { No. of hamsters } \\
\text { cured }\end{array}$ & $\begin{array}{l}\text { Mean worm } \\
\text { burden (SD) }\end{array}$ & $\begin{array}{l}\text { Total worm burden } \\
\text { reduction (\%) }\end{array}$ & Kruskal-Wallis \\
\hline Control & - & 6 & 0 & $10.8(3.1)$ & - & - \\
Tribendimidine & 400 & 5 & 0 & $4.0(4.5)$ & 63.0 & 3.76 \\
\hline
\end{tabular}

SD Standard deviation

summarized in Table 1. The mean worm burden observed in untreated control hamsters was 10.8 worms (standard deviation $[\mathrm{SD}]=3.1$ worms). After a single $400-\mathrm{mg} / \mathrm{kg}$ oral dose of tribendimidine, a mean worm burden of 4.0 worms ( $\mathrm{SD}=4.5$ worms) was found, which translated to a worm burden reduction of $63.0 \%(\mathrm{KW}=3.76, P=0.052)$.

\section{SEM observations of control $O$. viverrini}

The tegument of adult $O$. viverrini recovered from untreated hamsters and also incubated in vitro appeared normal. Figure 1 depicts the dorsal surface of an adult $O$. viverrini, and Fig. 2 shows the oral sucker of the same specimen.

In vitro SEM observations

Opisthorchis viverrini exposed to tribendimidine at a concentration of $10 \mu \mathrm{g} / \mathrm{ml}$ contracted immediately. Within $4 \mathrm{~h}$ of incubation in a culture medium containing $10 \mu \mathrm{g} / \mathrm{ml}$ tribendimidine all $O$. viverrini had died. SEM analysis from these flukes revealed an intact tegument (Fig. 3). The only difference we observed to untreated $O$. viverrini was a slightly reduced opening of the oral sucker (Fig. 4), blebbing on the tegumental surface (Fig. 3), and blebbing and swelling near the oral sucker (Fig. 4).

In vivo SEM observations

Twenty-four hours after administration of a single $400-\mathrm{mg} / \mathrm{kg}$ dose of tribendimidine to $O$. viverrini-infected hamsters, 10 flukes were recovered from the bile ducts. These flukes showed normal mobility. SEM observations of the flukes, however, revealed substantial tegumental damage both on the ventral and dorsal portions of the flukes as well as on the anterior and posterior regions of the trematodes. There was a reduced opening of the oral sucker (Fig. 5). Extensive sloughing was detected on the entire ventral and dorsal surfaces of most flukes examined (Figs. 6, 7 and 8). Numerous blebs were visible near the ventral sucker and the ventral and dorsal surfaces (Figs. 6, 7 and 8). Severe tegumental damage was also seen in the tail regions of the trematodes (Fig. 8).

Forty-eight hours post-treatment, nine $O$. viverrini could be recovered from the one hamster killed at this time point
Fig. 1 Opisthorchis viverrini obtained from an untreated control hamster

Fig. 2 Oral sucker $(O S)$ and papillae $(P)$ of a control

$O$. viverrini

Fig. 3 SEM of adult $O$. viverrini after exposure to $10 \mu \mathrm{g} / \mathrm{ml}$ tribendimidine in vitro. Ventral tegument with ventral sucker $(V S)$ of $O$. viverrini. Light blebbing $(B)$ visible

Fig. 4 SEM of adult $O$. viverrini after exposure to $10 \mu \mathrm{g} / \mathrm{ml}$ tribendimidine in vitro. Reduced opening, swelling $(S W)$ and blebbing $(B)$ of oral sucker $(O S)$
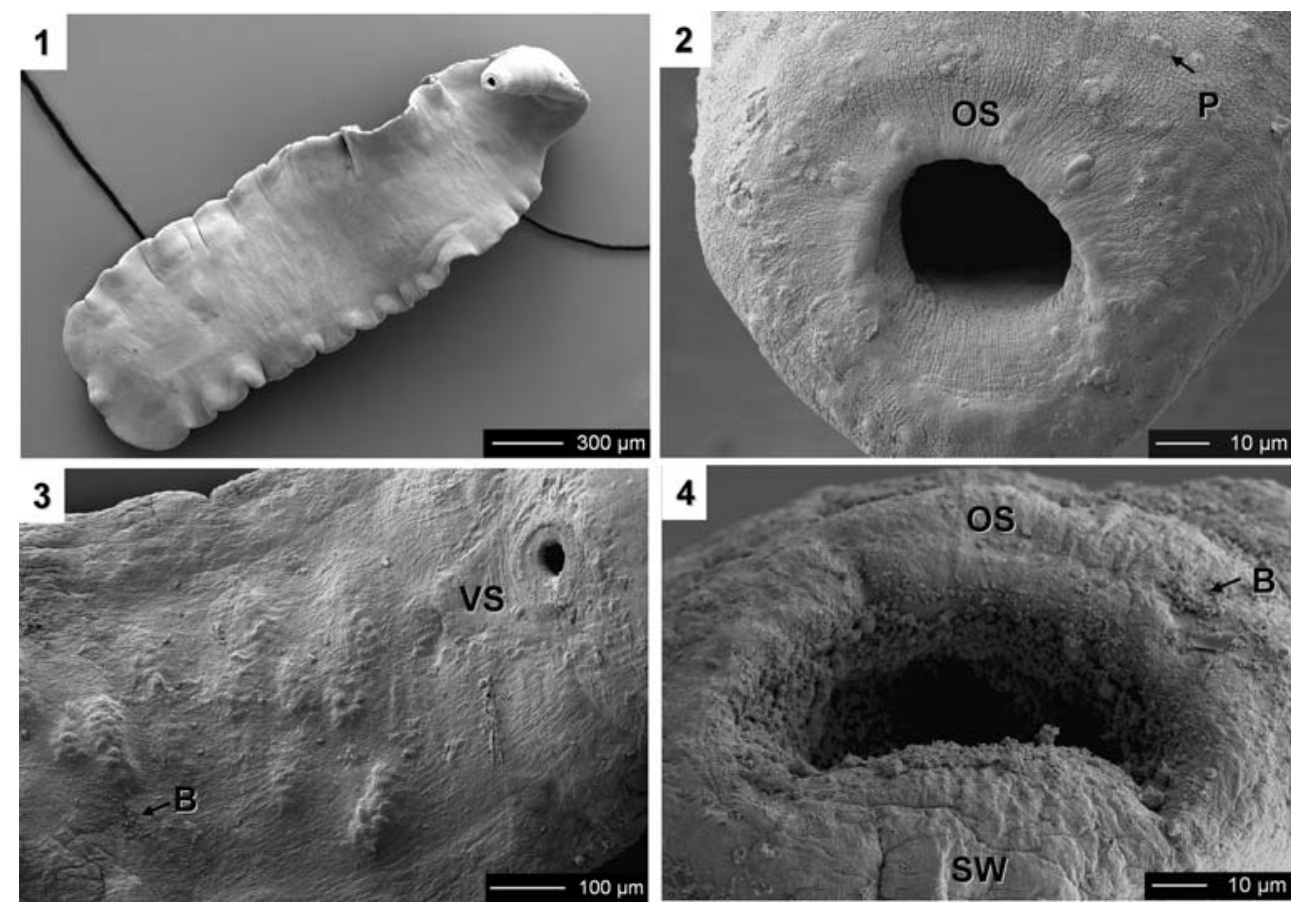
Fig. 5 SEM of adult $O$. viverrini $24 \mathrm{~h}$ post-treatment with a single $400-\mathrm{mg} / \mathrm{kg}$ oral dose of tribendimidine. Closure of the oral sucker $(O S)$

Fig. 6 SEM of adult $O$. viverrini $24 \mathrm{~h}$ post-treatment with a single $400-\mathrm{mg} / \mathrm{kg}$ oral dose of tribendimidine. Sloughing $(S)$ and blebbing $(B)$ near the ventral sucker $(v s)$

Fig. 7 SEM of adult $O$. viverrini $24 \mathrm{~h}$ post-treatment with a single $400-\mathrm{mg} / \mathrm{kg}$ oral dose of tribendimidine. Sloughing $(S)$ and blebbing $(B)$ near the ventral sucker $(v s)$

Fig. 8 SEM of adult $O$. viverrini $24 \mathrm{~h}$ post-treatment with a single $400-\mathrm{mg} / \mathrm{kg}$ oral dose of tribendimidine. Blebbing $(B)$ and sloughing $(S)$ on the tail region
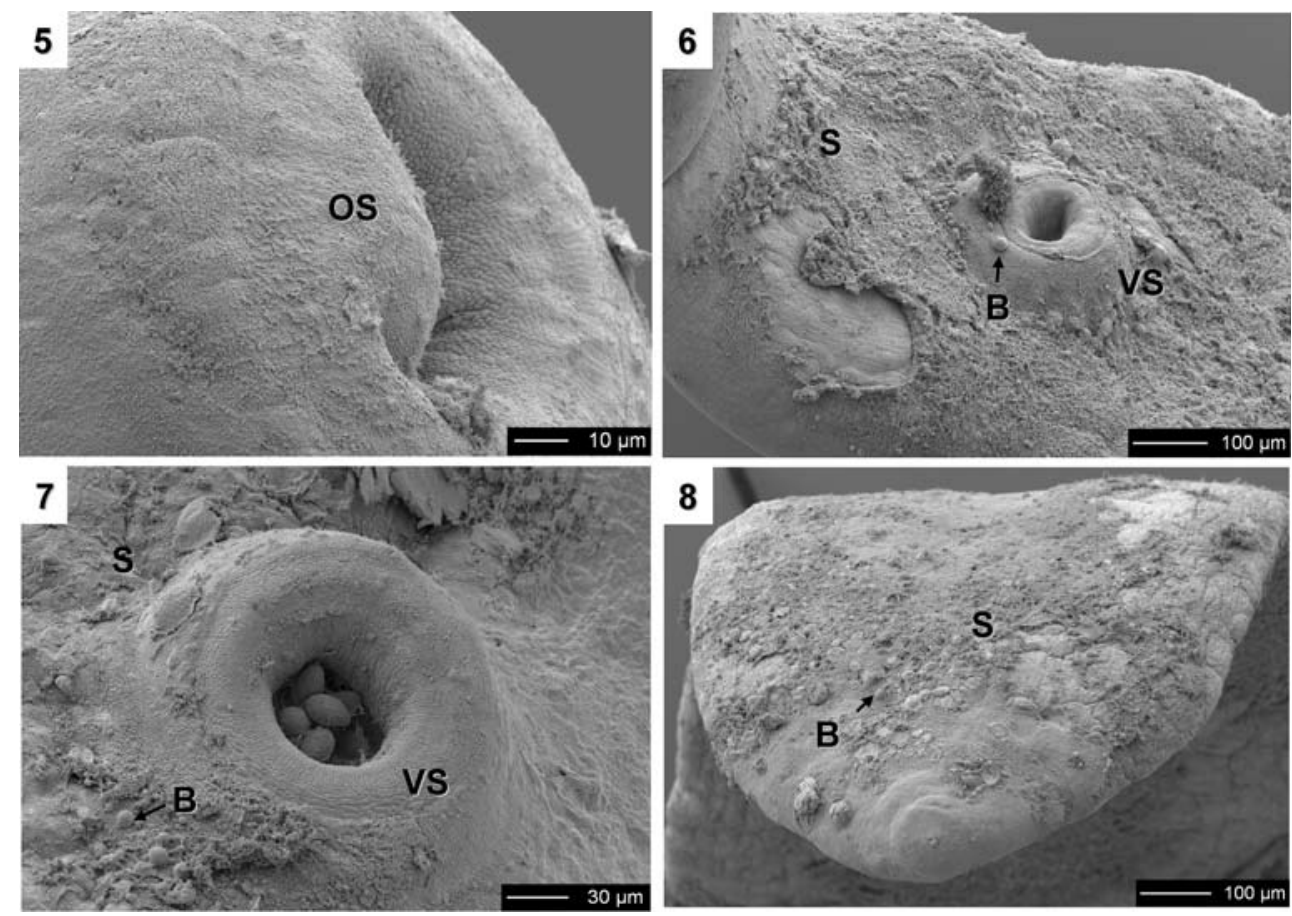

after tribendimidine administration. The flukes showed reduced mobility. SEM observations of these flukes showed similar tegumental disruption as during the previous time point, characterized by extensive sloughing of the tegument (no picture shown). Moreover, several trematodes showed a complete closure of their oral sucker (Fig. 9). In one specimen, extensive furrowing was also observed on the dorsal surface (Fig. 10).
Seventy-two hours after tribendimidine administration, we were able to extract $10 \mathrm{O}$. viverrini from the bile duct of the one hamster designated at this time point. All $O$. viverrini were alive, but showed reduced mobility. Tegumental damage was pronounced, and prominent findings included the sloughing of the tegumental surfaces (Figs. 11 and 12). Actually, the whole flukes were covered in blebs (Figs. 11 and 12).
Fig. 9 SEM of adult $O$. viverrini $48 \mathrm{~h}$ post-treatment with a single $400-\mathrm{mg} / \mathrm{kg}$ oral dose of tribendimidine. Closure of the oral sucker $(O S)$ and blebbing $(B)$

Fig. 10 SEM of adult $O$. viverrini $48 \mathrm{~h}$ post-treatment with a single $400-\mathrm{mg} / \mathrm{kg}$ oral dose of tribendimidine. Blebbing $(B)$ and furrowing $(F)$ on the dorsal surface

Fig. 11 SEM of adult O. viverrini $72 \mathrm{~h}$ post-treatment with a single $400-\mathrm{mg} / \mathrm{kg}$ oral dose of tribendimidine. Extensive sloughing $(S)$ and blebbing $(B)$ near the ventral sucker $(V S)$ Fig. 12 SEM of adult O. viverrini $72 \mathrm{~h}$ post-treatment with a single $400 \mathrm{mg} / \mathrm{kg}$ oral dose of tribendimidine. Blebbing $(B)$ and sloughing $(S)$ on the dorsal surface
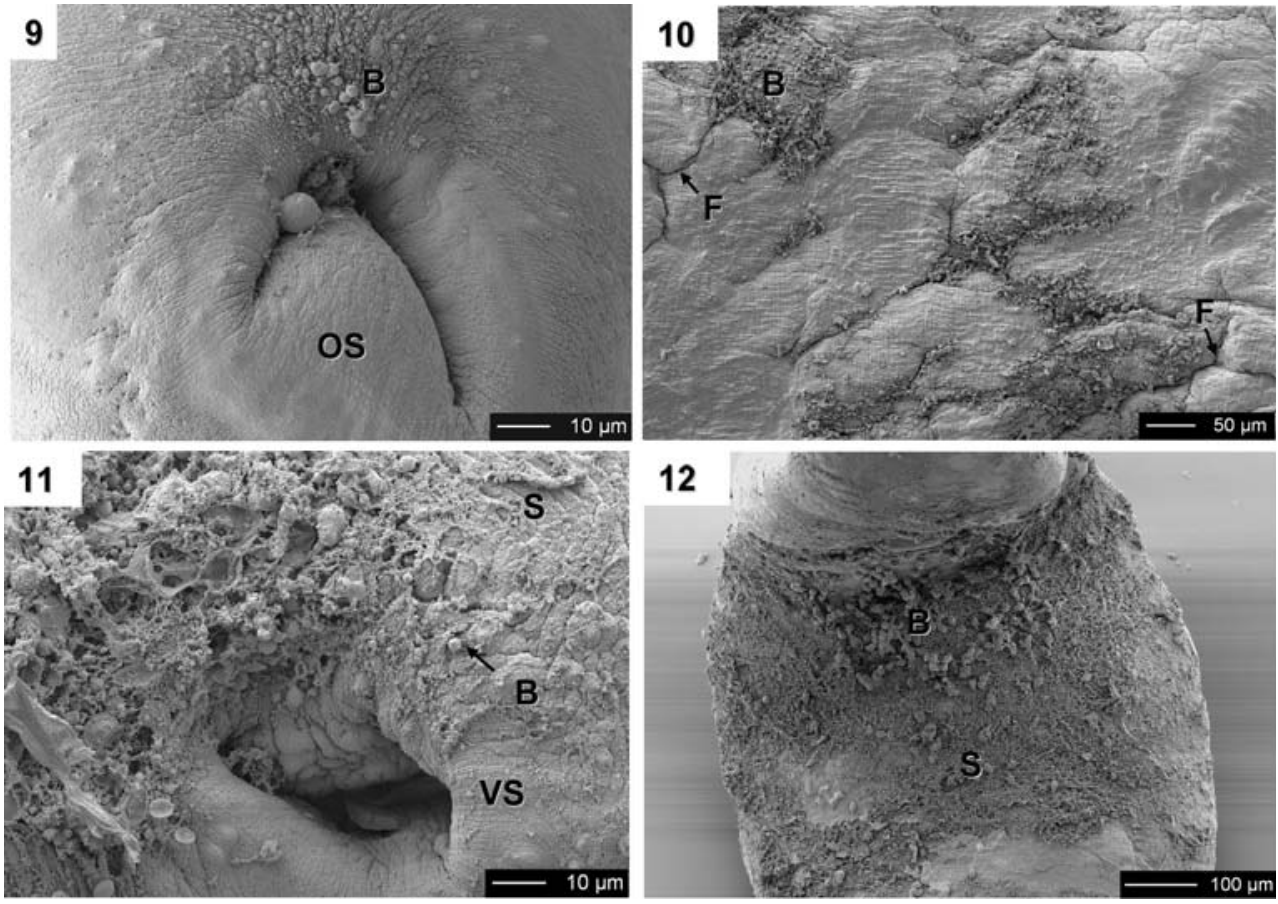
Ninety-six hours after treatment, the majority of $O$. viverrini had been expelled and only two $O$. viverrini could be obtained from the bile duct of the one treated hamster that was killed at this time point. One of these flukes was dead. In view of only one specimen alive at this time point post-treatment, no SEM analysis was carried out.

\section{Discussion}

To our knowledge, this is the first temporal examination of tegumental alterations on the surface of $O$. viverrini recovered from hamsters following administration of a single $400-\mathrm{mg} / \mathrm{kg}$ oral dose of tribendimidine, and after in vitro exposure of $O$. viverrini to a medium containing $10 \mu \mathrm{g} / \mathrm{ml}$ tribendimidine. We employed SEM as with our preceding work focusing on different food-borne trematodes and investigating the effect of the artemisinins, synthetic trioxolanes, and tribendimidine (Keiser et al. 2006c; Keiser and Morson 2008).

As discovery and development of drugs against opisthorchiasis in particular, and food-borne trematodiasis in general, has been sparse in the past decades, only few studies have elucidated the effect of drugs on the tegument of $O$. viverrini and other food-borne trematodes. Blebbing, swelling, and sloughing has been observed in $O$. viverrini that had been exposed to the current drug of choice, i.e., praziquantel, both in vitro and in vivo (Becker et al. 1981; Mehlhorn et al. 1983; Sirisinha et al. 1984; Apinhasmit and Sobhon 1996). In addition, sloughing and tegumental lesions were documented in $O$. viverrini specimen following multiple doses of the experimental drug amoscanate (Sobhon et al. 1986). Severe tegumental disruption as sloughing, furrowing, and blebbing was also observed in our study after administration of tribendimidine to $O$. viverrini-infected hamsters. These tegumental alterations were already evident $24 \mathrm{~h}$ post-treatment.

Interestingly, tegumental changes did not increase further in severity. An important finding was a reduced opening of the oral sucker of $O$. viverrini at $24 \mathrm{~h}$ and a complete closing at $48 \mathrm{~h}$ post-treatment. This finding corroborates with a recent report that elucidated the damage on the tegument of the intestinal fluke E. caproni after tribendimidine administration to infected mice (Keiser et al. 2006c). However, in contrast to the tegument of E. caproni, where mainly the ventral portion and the apical cone region of the worms were affected after administration of tribendimidine, no clear dorsal-ventral and anterior-posterior difference in tegument disruption was evident when $O$. viverrini were examined by SEM. Furthermore, while tribendimidine showed a rapid onset of action on E. capronithe majority of E. caproni had been expelled $8 \mathrm{~h}$ posttreatment with tribendimidine (Keiser et al. 2006c)-
O. viverrini were still alive $72 \mathrm{~h}$ (and one even at $96 \mathrm{~h}$ ) post-treatment. Differences in the susceptibility between these two trematode species, the location of the parasite in the host, and the different rodent models used (mouse in the case of $E$. caproni and hamster in $O$. viverrini) might explain these differences.

A quick drug action was also observed when $O$. viverrini were incubated in a cultivation medium containing tribendimidine. The differences between the fast drug action on $O$. viverrini in vitro and the slower action in vivo might be explained by the extensive biotransformation of the drug. In fact, it has been demonstrated that tribendimidine is rapidly metabolized to $p$-(1-dimethlyamino ethylimino) aniline.

Interestingly, in this study we observed a somewhat lower worm burden reduction (63.0\%) after a single $400-\mathrm{mg} / \mathrm{kg}$ oral dose of tribendimidine was administered to $O$. viverriniinfected hamsters when compared to our previous study, where we found a worm burden reduction of $95.7 \%$ at the same single dose of tribendimidine. This finding might be explained by inter-individual differences in drug absorption, gender (female vs. male hamsters, which had been used in the previous study) and higher infection intensity (mean worm burden of 10.8 worms per hamster) in this study, when compared to our previous investigation (mean worm burden: 5.8 worms per hamster). Further studies are necessary to elucidate the relationship between infection intensities and the opisthorchicidal effect of tribendimidine and other potential trematocidal drugs.

In conclusion, our study confirms previous results that tribendimidine displays interesting trematocidal activity in vitro and in vivo (Keiser et al. 2006b) and that the tegument of trematodes is a potential drug target of this anthelminthic. Further studies should also assess the damage to internal tissues and organs by means of transmission electron microscopy. In addition, the efficacy, tegumental alterations, and damage to internal tissues and organs of immature flukes due to tribendimidine administration remain to be investigated.

Acknowledgments We thank Mr. Gianni Morson at the Centre for Microscopy, University of Basel for expert help with the SEM studies. J. Keiser (project no. PPOOA-114941), J. Utzinger (project no. PPOOB-102883) and P. Odermatt (project no. NF3270B0-110020) are grateful to the Swiss National Science Foundation for financial support.

\section{References}

Apinhasmit W, Sobhon P (1996) Opisthorchis viverrini: effect of praziquantel on the adult tegument. Southeast Asian J Trop Med Public Health 27:304-311

Becker B, Mehlhorn H, Andrews P, Thomas H (1981) Ultrastructural investigations on the effect of praziquantel on the tegument of five species of cestodes. Z Parasitenkd 64:257-269 
Haswell-Elkins M, Levri E (2003) Food-borne trematodes. In: Cook GC, Zumla AL (eds) Manson's tropical diseases, 21st edn. Saunders, London, pp. 1471-1486

Keiser J, Utzinger J (2007a) Advances in the discovery and development of novel trematocidal drugs. Exp Opin Drug Discov 2(Suppl 1):S9-S23

Keiser J, Utzinger J (2007b) Food-borne trematodiasis: current chemotherapy and advances with artemisinins and synthetic trioxolanes. Trends Parasitol 23:555-562

Keiser J, Morson G (2008) Fasciola hepatica: tegumental alterations in adult flukes following in vitro and in vivo administration of artesunate and artemether. Exp Parasitol 118:228-237

Keiser J, Xiao SH, Jian X, Chang ZS, Odermatt P, Tesana S, Tanner M, Utzinger J (2006a) Effect of artesunate and artemether against Clonorchis sinensis and Opisthorchis viverrini in rodent models. Int J Antimicrob Agents 28:370-373

Keiser J, Xiao SH, Tanner M, Utzinger J (2006b) Artesunate and artemether are effective fasciolicides in the rat model and in vitro. J Antimicrob Chemother 57:1139-1145

Keiser J, Xiao SH, Utzinger J (2006c) Effect of tribendimidine on adult Echinostoma caproni harbored in mice, including scanning electron microscopic observations. J Parasitol 92:858-862

Keiser J, Thiemann K, Endriss Y, Utzinger J (2008) Strongyloides ratti: in vitro and in vivo activity of tribendimidine. PLoS Negl Trop Dis 2:e136
Mehlhorn H, Kojima S, Rim HJ, Ruenwongsa P, Andrews P, Thomas H, Bunnag B (1983) Ultrastructural investigations on the effects of praziquantel on human trematodes from Asia: Clonorchis sinensis, Metagonimus yokogawai, Opisthorchis viverrini, Paragonimus westermani and Schistosoma japonicum. Arzneimittelforschung 33:91-98

Nwaka S, Hudson A (2006) Innovative lead discovery strategies for tropical diseases. Nat Rev Drug Discov 5:941-955

Ren HN, Cheng BZ, Zhuang ZN (1987) Experimental therapeutic efficacy of a new anti-hookworm drug-tribendimidine. Chin J Parasitol Parasit Dis 5:262-264 (in Chinese)

Sirisinha S, Puengtomwatanakul S, Sobhon P, Saitongdee P, Wongpayabal P, Mitranonde V, Radomyos P, Bunnag D, Harinasuta T (1984) Alterations of the surface tegument of Opisthorchis viverrini exposed to praziquantel in vitro and in vivo. Southeast Asian J Trop Med Public Health 15:95-103

Sobhon P, Wanichanond C, Saitongdee P, Koonchornboon T, Bubphaniroj P, Upatham ES, Puengtomwatanakul S, Sirisinha S (1986) Scanning electron microscopic study of Opisthorchis viverrini tegument and its alterations induced by amoscanate. Int J Parasitol 16:19-26

Sripa B (2003) Pathobiology of opisthorchiasis: an update. Acta Trop 88:209-220

Xiao SH, Wu HM, Tanner M, Utzinger J, Wang C (2005) Tribendimidine: a promising, safe and broad-spectrum anthelminthic agent from China. Acta Trop 94:1-14 